\title{
Session III: Breast
}

\section{Breast imaging for risk assessment, cancer preven- tion, and early detection}

\author{
Carol J. Fabian \\ University of Kansas Cancer Center, Clinical Oncolo- \\ gy, Kansas City, KS 66160, USA
}

Epidemiologic models such as the Gail model are validated for populations but have suboptimal discriminatory accuracy, making it difficult to advise women regarding the need or urgency for prevention drugs or prophylactic surgery. A number of reversible risk biomarkers have been established for use in risk assessment [1]. The two most powerful are mammographic breast density and evidence of precancerous changes in diagnostic or nondiagnostic tissue samples. Women with $>75 \%$ breast density have a five-fold increase in risk (for at least the next five years) relative to those with essentially no breast density [2]. Women with histologic or cytologic evidence of atypia have a fivefold increase in cancer risk over the next 5-10 years compared with those with no evidence of atypia [3,4]. Women with atypical hyperplasia tend to have higher mammographic breast densities (and vice versa), but the two biomarkers may have independent predictive significance. Both mammographic breast density assessed by breast imaging, reporting, and data system (BIRADS) and cytomorphologic atypia (from samples obtained by random periareolar fine-needle aspiration of high-risk women), when added to the Gail model, improve discriminatory accuracy as assessed by the concordance statistic [5,6]. With the use of BIRADS breast density categorization, however, this improvement is very modest. Especially for young high-risk women, an inexpensive imaging technique is needed which would avoid the use of radiation, accurately quantitate the portion of the breast encompassed by stroma and epithelium, and if possible, permit detection of early breast cancers. Breast spectroscopy is attractive in this regard.

Breast-specific reversible risk biomarkers such as mammographic density, breast cytomorphology, and proliferation may serve both to help select appropriate candidates for prevention and monitor response to standard prevention therapy, or as part of a clinical trial to evaluate a new agent [7].

Tamoxifen, a standard prevention drug, substantially reduces mammographic density in women younger than 45 but not in women older than 55 [8]. It is not clear at present whether any drug likely to be effective in primary prevention substantially reduces density in postmenopausal women. Mammographic density may be most useful as a response biomarker in preand perimenopausal women. At present, it is unclear whether any drug, including selective estrogen receptor modulators or aromatase inhibitors, substantially alters tissue morphology after 6-12 months of treatment, the interval commonly used in Phase II prevention trials. This is in part due to the difficulty in quantitating the amount or volume of benign breast disease. A woman is considered to have atypical hyperplasia if $1 \%$ or $70 \%$ of epithelial cells captured in a tissue sampling procedure are atypical.

Proliferation in areas of hyperplasia has been identified as a risk factor in a cross-sectional study [9] and is currently being used as a response biomarker in Phase II trials [7]. Tamoxifen is known to reduce proliferation in benign breast disease and breast cancer. Further, in cancer treatment trials, reduction in proliferation predicts clinical response (reviewed in [7]).

If spectral imaging could be performed inexpensively, it would be ideal for assessing a response to an intervention, as no radiation is involved. Mammography and sonography are currently the two primary modalities for early detection. Screening breast MRI is more sensitive than mammography but, given the expense of imaging, is generally reserved for women from hereditary breast cancer families.

\section{References}

[1] C.J. Fabian, B.F. Kimler, M.S. Mayo and S.A. Khan, Breasttissue sampling for risk assessment and prevention, Endocr Relat Cancer 12 (2005), 185-213. 
[2] N.F. Boyd, J.W. Byng, R.A. Jong et al., Quantitative classification of mammographic densities and breast cancer risk: results from the Canadian National Breast Screening Study, J Natl Cancer Inst 87 (1995), 670-675.

[3] L.C. Hartmann, T.A. Sellers, M.H. Frost et al., Benign breast disease and the risk of breast cancer, $N$ Engl J Med 353 (2005), 229-237.

[4] C.J. Fabian, B.F. Kimler, C.M. Zalles et al., Short-term breast cancer prediction by random periareolar fine-needle aspiration cytology and the Gail risk model, J Natl Cancer Inst 92 (2000), 1217-1227.

[5] M.S. Mayo, B.F. Kimler and C.J. Fabian, Evaluation of models for the prediction of breast cancer development in women at high risk, J Applied Res 1 (2001), 37-44.

[6] J.A. Tice, S.R. Cummings, E. Ziv and K. Kerlikowske, Mammographic breast density and the Gail model for breast cancer risk prediction in a screening population, Breast Cancer Res Treat 94 (2005), 115-122.

[7] C.J. Fabian, B.F. Kimler, M.S. Mayo, W.E. Grizzle, S. Masood and G. Ursin, Clinical approaches to discovering and testing new breast cancer prevention drugs, in: Cancer Chemoprevention, Vol. 2; Strategies for Cancer Chemoprevention, G.J Kelloff, E.T. Hawk and C.C. Sigman, eds, Totowa, New Jersey: Humana Press, 2005, pp. 213-238.

[8] J. Cuzick, J. Warwick, E. Pinney, R.M. Warren and S.W. Duffy, Tamoxifen and breast density in women at increased risk of breast cancer, J Natl Cancer Inst 96 (2004), 621-628.

[9] A.M. Shaaban, J.P. Sloane, C.R. West and C.S. Foster, Breast cancer risk in usual ductal hyperplasia is defined by estrogen receptor-alpha and Ki-67 expression, Am J Pathol 160 (2002), 597-604.

\section{The role of ultrasound in tumor imaging and image- guided therapy}

\section{Katherine Ferrara}

University of California, Davis, Department of Biomedical Engineering, Davis, CA 95616, USA

Ultrasound imaging is frequently used in the differential diagnosis of suspicious lesions and as a means to guide biopsy. With the addition of a contrast agent, angiogenic breast lesions on the order of a few millimeters can be detected in preclinical studies. Serial studies with and without anti-angiogenic therapy indicate that ultrasound-based estimates of perfused tumor volume are highly correlated with CT-based estimates of tumor volume and measurements of viable tumor based on histology. Ultrasound contrast agents targeted to $\forall \mathrm{v} \exists 3$ have been shown to adhere to the endothelium in angiogenic tumors and may further improve the detection and characterization of small lesions. In addition to its application in imaging, the use of ultrasound to ablate breast, ovarian, liver, prostate, and brain lesions shows great promise. Local delivery of a drug or gene can also be facilitated.
Near-infrared breast imaging: Spectral measurements for tumor oximetry ${ }^{1}$

Sergio Fantini, Ning Liu and Angelo Sassaroli

Tufts University, Department of Biomedical Engineering, Medford, MA 02155, USA

Optical mammography, a non-invasive imaging modality, is mostly sensitive to the concentration and oxygen saturation of hemoglobin within the breast, and also provides information on spatial distribution of lipids and water concentration. At right are twodimensional projection images of the left breast of a 53-year old patient; the top image is a spatial-secondderivative image (at $690 \mathrm{~nm}$ ) that enhances the visibility of blood vessels, while the bottom image is an oxygenation image based on optical data at four wavelengths $(690,750,788$, and $856 \mathrm{~nm})$ that takes advantage of the dependence of the absorption spectrum of hemoglobin on its oxygen saturation. A $3 \mathrm{~cm}$ invasive ductal carcinoma (indicated by the arrow) is visible as an area of high blood vessel density (top image) and low oxygenation (bottom image).

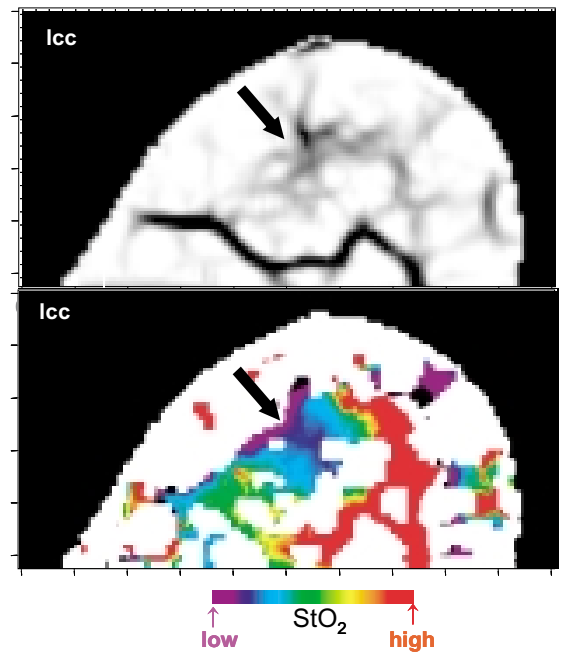

To enhance the spatial information of optical mammograms, we are developing a pseudo-phased-array approach that uses a planar array of sources (on the illumination side) and a planar array of detectors (on the collection side). We have shown that this approach enhances the spatial resolution and achieves depth discrimination in diffuse optical imaging.

\footnotetext{
${ }^{1}$ This research is supported by the National Institutes of Health (Grant No. CA95885).
} 
To obtain more quantitative oxygenation images, we have introduced a novel method based on measurement of a continuum optical spectrum to identify two optimal wavelengths (which depend on the oxygenation level of the tumor) to measure tumor oxygenation. Once an optimal wavelength pair is identified for each case, the resulting measurement of tumor oxygenation is expected to be largely independent of unknown factors such as the tumor size, shape, and depth within the breast.

\section{Magnetic resonance image-guided breast cancer therapy}

Mitchell D. Schnall

University of Pennsylvania, Philadelphia, PA 19123, USA

There is expanding use of MRI to evaluate the extent of primary breast cancer in order to plan appropriate therapy. Single and multicenter data clearly establish that contrast-enhanced MRI is the best technique to detect multicentric and multifocal disease. Although its clinical role in evaluating women for traditional breast conservation therapy is controversial, MRI will clearly assist in the development of minimally invasive techniques targeted at early disease. In addition to identifying candidates for minimally invasive breast cancer treatment by excluding multifocal and multicentric cancer, MRI can also be applied to target definition and direct image guidance. As a functional imaging modality, MRI can also be involved in response assessment. Further, MRI can also directly monitor the tissue temperature to ensure adequate treatment. The integration of focused ultrasound into the MRI environment offers an exciting opportunity to leverage the features of MRI cited above to deliver noninvasive ablative therapy. 